A N N A L E S Annales de Bretagne et des Pays de l'Ouest

\title{
L'expérience corsaire : entre opportunités et illusions
}

Les relations entre la course et les gens de mer du Pays basque, de 1725 à 1755

Lucas Bossebœuf

\section{(2) OpenEdition}

Journals

Édition électronique

URL : http://journals.openedition.org/abpo/3832

DOI : $10.4000 /$ abpo.3832

ISSN : 2108-6443

Éditeur

Presses universitaires de Rennes

Édition imprimée

Date de publication : 12 juillet 2018

Pagination : 89-105

ISBN : 978-2-7535-7604-9

ISSN : 0399-0826

Référence électronique

Lucas Bossebœuf, «L'expérience corsaire : entre opportunités et illusions », Annales de Bretagne et des Pays de l'Ouest [En ligne], 125-2 | 2018, mis en ligne le 12 juillet 2020, consulté le 06 janvier 2021. URL : http://journals.openedition.org/abpo/3832 ; DOI : https://doi.org/10.4000/abpo.3832 


\title{
L'expérience corsaire : entre opportunités et illusions
}

\section{Les relations entre la course et les gens de mer du Pays basque, de 1725 à 1755}

\author{
Lucas BossebeEuF \\ Master 2 histoire, université de Poitiers (direction : Thierry Sauzeau)
}

"Les braves et aventureux marins de Bayonne et du Pays basque méritaient de sortir de l'oubli dans lequel ils étaient déjà plongés ${ }^{1}$. " Pour exalter leurs figures romanesques, l'historien bayonnais Édouard Ducéré (1849-1910) donne à son récit des corsaires bayonnais l'allure d'une histoire bataille. Il restitue ainsi la biographie de plusieurs figures basques, accompagnées de leurs prouesses presque chevaleresques. Néanmoins, au-delà de ce récit, d'autres facettes de la vie des marins de la course sont dévoilées : la mort, la promotion professionnelle, ainsi que l'invalidité ou encore l'abandon de cette activité pour les plus traumatisés, sont évoqués ${ }^{2}$ en arrière-plan de récits d'abordages et de dangereuses manœuvres.

La bataille de la Hougue, en 1692, a démontré les limites de la guerre d'escadre, coûteuse et risquée pour l'État. Aussi, en France, la Couronne encourage-t-elle les armateurs à se lancer dans la course, encadrée par la grande ordonnance de la Marine $(1681)^{3}$. Pour prétendre chasser l'ennemi en mer, les armateurs doivent impérativement se munir d'une lettre de marque, délivrée par l'Amirauté, sous peine d'être convaincus de piraterie. Il convient ensuite de faire un maximum de prises ennemies, dont la nationalité était préalablement définie dans la lettre. Si la Couronne compte réduire les coûts de la guerre maritime et gêner les échanges entre l'ennemi

1. DuCÉRÉ, Édouard, Histoire maritime de Bayonne. Les corsaires sous l'Ancien Régime, Bayonne, E. Hourquet, 1895, np.

2. Se référer à la lettre rédigée par le capitaine corsaire bayonnais, Pierre Dolacre, publié dans DucÉRÉ, Édouard, ibid., p. 276-277.

3. LE BOUËDEC, Gérard, Les activités maritimes et sociétés littorales de l'Europe atlantique : 1690-1790, Paris, Armand Colin, 1997, p. 221. Et pour plus de précisions quant à la contribution de Maurepas à la marine française, se référer à VERGE-FRANCESCHI, Michel, La marine française au XVIII siècle : guerres, administration, exploration, Paris, SEDES, 1996, p. 84-101. 
anglais et ses colonies, les entrepreneurs de cette guerre privée, eux, envisagent la course comme un moyen de limiter les pertes financières causées par l'asphyxie du commerce. Durant la guerre de Succession d'Autriche (1744-1748), bien que les Anglais parviennent rapidement à paralyser la façade atlantique, quelques ports français font preuve de résistance, et tout particulièrement Bayonne qui tire ainsi son épingle du jeu ${ }^{4}$.

Bayonne est, à l'époque moderne, un port de pêche et de commerce d'environ 15000 habitants $^{5}$, qui émerge dans le paysage maritime français ${ }^{6}$. Son ascension tient à sa proximité avec l'Espagne qui fait d'elle une ville à surveiller et à protéger. Éloignés du pouvoir central, isolés des autres grandes villes et limités par un arrière-pays pauvre, les Bayonnais tournent le dos aux montagnes pour regarder vers la mer, favorisés dans leurs entreprises par la navigabilité de l'Adour. Exclu de la liste des ports privilégiés pour faire le grand commerce, ce port se cantonne au cabotage et à la pêche : les trajets aux Antilles y représentent 2 à $3 \%$ des armements jusqu'au milieu du XVIII ${ }^{\mathrm{e}}$ siècle ${ }^{7}$. Toutefois, l'amélioration des relations entre les royaumes de France et d'Espagne lui apporte au XVIII ${ }^{\mathrm{e}}$ siècle des déceptions. Bayonne perd un peu de sa valeur géostratégique et est frappée par plusieurs crises économiques. Seules les guerres inversent un peu la tendance, offrant aux armateurs et négociants l'opportunité de prendre le risque de reconvertir en navires corsaires leurs forts navires de grand cabotage, désarmés en temps de guerre. À partir de la guerre de Succession d'Autriche, Bayonne prend place parmi les trois ports corsaires les plus actifs du royaume ${ }^{8}$.

La course basque est un objet d'histoire bien connu, toutefois sa dimension sociale reste riche de questions. En effet, si l'on s'attarde sur celle du rapport entre la course et les gens de mer, pour examiner ses retombées, on remarque qu'elle a intrigué nombre d'historiens français, comme en témoignent les ouvrages d'André Lespagnol pour Saint-Malo ${ }^{9}$ et de Jacques Péret pour le littoral atlantique ${ }^{10}$, mais aussi d'Alain Cabantous pour les marins de la Manche orientale ${ }^{11}$. Tous ont été unanimes pour affirmer que la course jouait un rôle d'accélérateur professionnel dans la vie des gens de mer. Cependant, ces recherches se sont focalisées sur les périodes de conflit, et la question des retombées de la guerre de course

4. PÉRET, Jacques, Les corsaires de l'Atlantique : de Louis XIV à Napoléon, La Crèche, Geste éditions, 2012, p. 25.

5. ACERRA, Martine, MEYER, Jean, La grande époque de la Marine à voile, Ouest France éd, 1995, p. 86.

6. PONTET, Josette, Histoire de Bayonne, Toulouse, Privat, 1991, p. 132-135.

7. Ibid., p. 140.

8. PÉRET, Jacques, Les corsaires..., op. cit., p. 25.

9. LESPAGNOL, André, La course malouine au temps de Louis XIV entre l'argent et la gloire, Rennes, PUR, 1996.

10. PÉRET, Jacques, Les corsaires..., op. cit.

11. CaBantous, Alain, Dix mille marins face à l'océan: les populations maritimes de Dunkerque au Havre aux XVIT et XVIII siècles, vers 1660-1794 : étude sociale, Paris, Publisud, 1991. 
sur la carrière professionnelle des marins, à long terme, reste ouverte à la recherche historique.

Pour contribuer à y répondre, nous avons organisé le suivi des équipages de navires corsaires, afin de reconstituer les trajectoires individuelles en amont et en aval de l'expérience corsaire de la guerre de Succession d'Autriche. Au cours de ce conflit, Bayonne arme 103 corsaires ${ }^{12}$ parmi lesquels nous avons finalement isolé trois campagnes - la Bellonne, l'Entreprenante et la Victoire - au regard de leurs résultats : succès, réussite honnête, échec. Identifiés à travers les rôles d'armement de ces trois navires corsaires, les Basques ont ensuite été repérés dans les registres matricules, conservés au Service Historique de la Défense de Rochefort, relatifs aux quartiers de Bayonne et de Saint-Jean-de-Luz sur la période 1725-1764. Une telle source, qui commande la chronologie, permet d'envisager des carrières depuis l'apprentissage jusqu'à l'âge adulte. Son étude prend alors la forme d'un suivi de cohorte : on retient les marins ayant débuté après 1725 et on les suit jusqu'à l'année de clôture du registre, 1764. À travers cette étude, c'est l'impact de la guerre de course sur la vie des gens de mer du Pays basque, autour de la guerre de Succession d'Autriche, qui peut être appréhendée. Après avoir identifié les origines des trois entreprises de course sélectionnées et de leur main-d'œuvre locale, nous avons la possibilité de travailler sur l'apprentissage et la formation en amont, sur l'impact direct de la course sur la vie professionnelle des 105 gens de mer basques impliqués, mais aussi d'envisager leurs parcours une fois les traités de paix d'Aix-la-Chapelle signés, et ce jusqu'à la veille de la guerre de Sept Ans (1755), pour ceux qui ont survécu jusque-là.

\section{Des marins et des navires pour la course (1725-1744)}

À l'annonce de l'entrée en guerre de l'Angleterre, en mars 1744, onze corsaires sont armés à Bayonne et ils sont vingt de plus l'année suivante $^{13}$. Tous sont alors soumis à cette " loterie corsaire " dépeinte par André Lespagnol, et dont l'issue est illustrée par les résultats mitigés de nos trois navires corsaires. À bord, la discipline et la bonne exécution des manœuvres dépendent exclusivement d'un groupe intermédiaire, celui des officiers mariniers. Ils transmettent à l'équipage les ordres établis au sein de l'état-major, réuni autour du capitaine. L'étude de la composition de ce groupe de spécialistes de l'encadrement des hommes devient alors très révélatrice de l'état des rapports humains et de l'efficacité des rapports hiérarchiques à bord ${ }^{14}$.

12. Pontet, Josette, "Les corsaires dans l'Amirauté de Bayonne au XVIII ${ }^{\mathrm{e}}$ siècle ", Itsas Memoria. Revista de Estudios Marítimos del País Vasco, 5, Untzi Museoa-Museo Naval, Donostia-San Sebastián, 2006, p. 309-324.

13. Arch. du Service historique de la Défense (SHD) de Rochefort, 13P8/12 et 13.

14. SAUZEAu Thierry, Les marins de la Seudre. Du sel charentais au sucre antillais, La Crèche, Geste éditions, 2005, op. cit., p. 145. 
Figure 1 - Les officiers mariniers de La Bellonne, entre 1725 et 1744

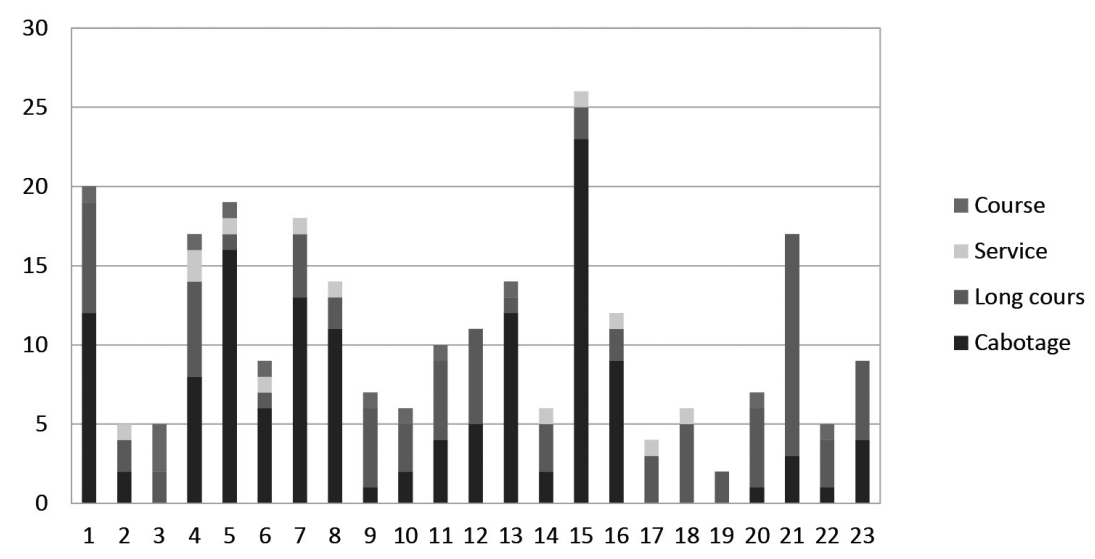

Ainsi, La Bellonne ${ }^{15}$, une frégate de 200 tonneaux, équipée de 20 canons et sortie de l'arsenal en 1744, est-elle bâtie et armée sous l'initiative de Dominique Labat - un important négociant originaire de Bayonne ${ }^{16}$ avant d'inaugurer sa première sortie en course le 5 février $1745^{17}$. Les 213 hommes enrôlés sous les ordres de Bertrand Piquessary affichent la plus jeune moyenne d'âge des trois corsaires étudiés, environ 23 ans. Autre caractéristique, les Basques représentent 70 \% de l'équipage. Avec une moyenne d'âge de 25 ans, les 23 Basques intégrés au groupe de la maistrance ont achevé leur apprentissage au cabotage et sont ensuite parvenus à intégrer plus ou moins rapidement les réseaux de navigation au long cours $^{18}$. Ils sont déjà habitués aux longues sorties en haute mer. De plus, la plupart témoigne d'expériences militaires, 19 d'entre eux ayant servi sur les navires du roi. Pour toutes ces raisons, l'équipage de La Bellonne apparaît comme le plus homogène des trois.

Cet atout n'est sans doute pas sans lien avec le fait que la loterie corsaire sourit à l'équipage. Dès le 28 février, il procède à la capture de La Bretagne de la Nouvelle-York ${ }^{19}$, avant d'effectuer la prise du Bond de Londres, le 26 mai, à hauteur de la pointe sud-ouest de l'Angleterre et du sud de l'Irlande ${ }^{20}$, zone de chasse privilégiée des corsaires français. Toutefois, si le montant de la revente rapporte près de 33250 livres aux armateurs associés, les profits n'équilibrent même pas la mise-hors qui s'élève à 110000 livres $^{21}$... En dépit de ce bilan mitigé, La Bellonne enre-

15. Arch. du SHD de Rochefort, 13P8/13, fol. 12.

16. Arch. dép. des Pyrénées-Atlantiques, 1J925.

17. Ibid., B8733.

18. Arch. du SHD de Rochefort, 13P6/4 et 15P6/9.

19. Arch. dép. des Pyrénées-Atlantiques, B8733.

20. Ibidem.

21. Ibidem. 
gistre néanmoins la seconde meilleure sortie corsaire à Bayonne pour les années 1744-1745, loin devant le fiasco de certaines croisières, à l'image du parcours de l'Entreprenante.

Figure. 2 - Les officiers mariniers de L'Entreprenante, entre 1725 et 1744

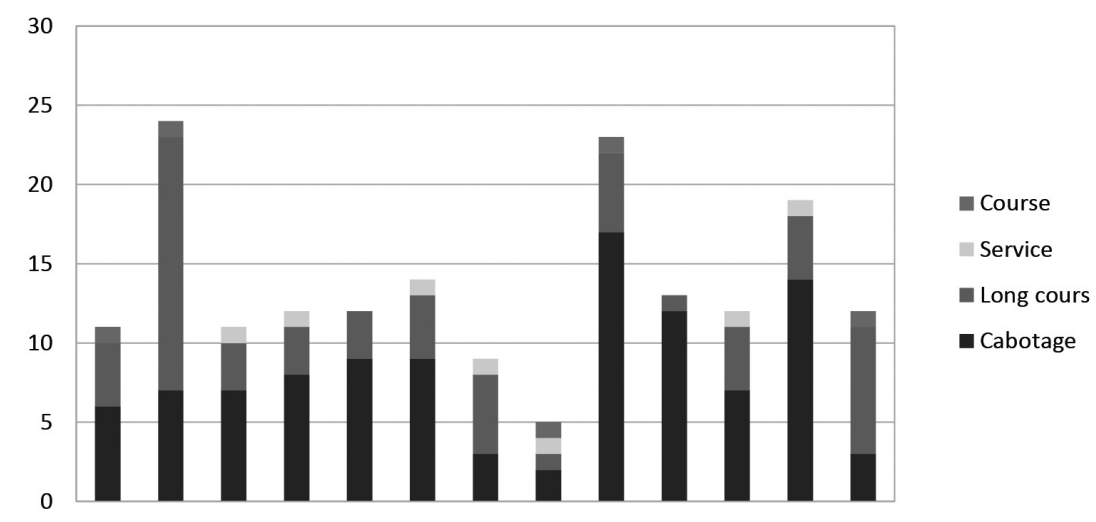

La campagne de L'Entreprenante ${ }^{22}$ démontre que la course est une activité risquée et aléatoire, à l'issue à la fois dramatique et banale. Construite à la demande de Jean Forestier et de Jean Dibiry, deux négociants et bourgeois de la ville de Bayonne, cette frégate jauge 120 tonneaux. Équipée de 12 canons et pierriers pour 116 hommes, elle part en course pour sa première campagne le 13 mars $1745^{23}$, sous le commandement de Raymond Dupuy. Après un début plutôt calme, la sortie ne porte véritablement ses fruits que le $1^{\mathrm{er}}$ juillet 1745 , date à laquelle le corsaire chasse La Marie de Porthampton ${ }^{24}$ au large des côtes anglaises. Au final, le maigre butin de 7100 livres récompense bien mal cinq mois de croisière pour les marins, et de patience pour les investisseurs restés sur la terre ferme. Cette prise semble constituer l'unique profit réalisé par l'équipage avant que, comme le mentionne le rôle d'armement sous la plume du commis aux écritures de l'Amirauté, le navire ne finisse "Pris par les Anglois. Le 25 juillet $1745^{25}$ ”. Cette courte mention rappelle qu'il ne faut alors qu'un pas pour passer du statut de prédateur à celui de proie, et accumuler les pertes.

La composition de l'équipage donne cependant quelques clés pour envisager les raisons d'un échec. En effet, avec une moyenne d'âge proche de 27 ans, l'équipage ne compte que $43 \%$ de marins du Pays basque qui sont minoritaires à la fois au sein de l'état-major (3 officiers sur 11) et parmi les matelots (7 matelots sur 47). Si les Basques sont en force parmi les

22. Arch. du SHD de Rochefort, 13P8/13, fol. 48.

23. Arch. dép. des Pyrénées-Atlantiques, B8733.

24. Ibidem.

25. Arch. du SHD de Rochefort, 13P8/13, fol. 48. 
officiers mariniers (16 marins sur 18), l'équipage est essentiellement composé de marins étrangers, auxquels s'ajoutent 40 volontaires, soit $30 \%$ du personnel enrôlé. En dépit de cette diversité, les parcours professionnels des marins basques, avant la course, sont sensiblement les mêmes que ceux de La Bellonne : tous ont déjà réalisé des voyages au long cours, la majorité d'entre eux compte au moins une campagne militaire; enfin, les sorties au cabotage dominent nettement la feuille de route de ces marins ${ }^{26}$.

L'équipage de L'Entreprenante se révèle donc plutôt hétérogène, mais sa composition semble sans lien direct avec sa capture par les Anglais, qui est plutôt à mettre sur le compte d'une rencontre malheureuse. C'est plutôt - pour reprendre l'expression de Jean de La Fontaine - « la loi du plus fort " qui est ici en cause, à la différence de ce que nous apprend le parcours de la Victoire ${ }^{27}$.

Figure 3 - Les officiers mariniers de La Victoire, entre 1725 et 1744

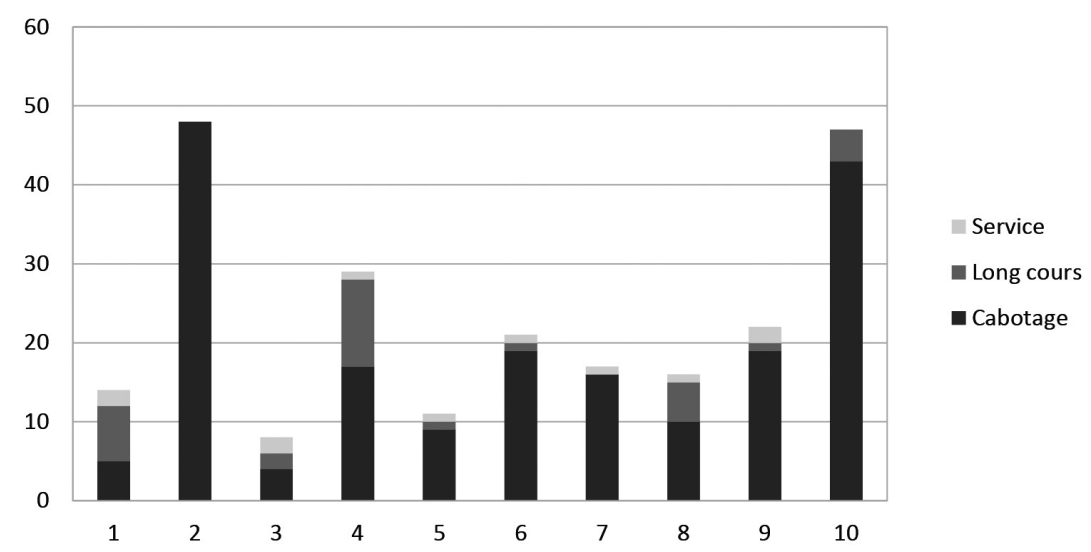

D'une jauge de 200 tonneaux, armé de 24 canons ainsi que de 8 pierriers pour parfaire une artillerie déjà complète, ce corsaire part en course le 27 juin $1744^{28}$. Armé par Jean Casaubon, il appartient à Jean Caulonque fils, et Antoine Duler est son commandant. Le parcours de ce navire se détache des deux autres, d'une part, par le nombre de prises - cinq au total - ramenées au port de Bayonne, pour une revente globale de 23675 livres, d'autre part, par la mutinerie qui paraît avoir éclaté à son bord après l'abordage litigieux de La Méditerranée de Bristol, le 28 août $1744^{29}$. L'acte est assez exceptionnel, dangereux et risqué, comme en témoignent le décès de cinq corsaires français et la blessure d'un marin débarqué à l'hôpital de Bayonne. Cet abordage semble malgré tout assez vain, compte tenu du

26. Arch. du SHD de Rochefort, 13P6/4.

27. Ibid., 13P8/12, fol. 56 .

28. Arch. dép. des Pyrénées-Atlantiques, B8733.

29. Ibidem. 
maigre butin dégagé : 5000 livres seulement... Ce médiocre rapport entre risque et bénéfice n'a-t-il pas causé des tensions entre les marins? En tout cas, le 11 septembre 1744, moins de deux semaines après l'abordage, cinq officiers corsaires sont congédiés. Bien que les sources ne nous permettent pas d'approfondir ce point, il semblerait que cette mesure ait été le prélude à un mouvement de mécontentement assez massif.

Après avoir appareillé, le corsaire tient la mer moins d'un mois avant que le capitaine ne semble contraint de rentrer au port. En effet, entre le 19 et le 21 octobre 1744, 87 marins - soit près d'un tiers de l'équipage désertent les rangs de l'expédition ${ }^{30}$. Si La Victoire s'en sort à première vue avec un profit satisfaisant, l'une des conséquences de cette possible rébellion est l'augmentation des dépenses. Marquée par une relâche forcée, la campagne de course est suspendue. Ajoutons à cet épisode un remplacement d'équipage dont le surcoût peut être estimé à 5318 livres ${ }^{31}$. Les présumés mutins étant exclusivement des étrangers au Pays basque, cette crise semble devoir être mise sur le compte d'un manque de cohésion de l'équipage. À l'image de leur capitaine, Antoine Duler, alors âgé de 61 ans, les corsaires basques de La Victoire sont également relativement âgés, 32 ans en moyenne. En outre, les étrangers, qui représentent plus de la moitié de l'équipage de L'Entreprenante (57 \%), en constituent ici les deux tiers (67\%). Le déséquilibre est un peu moindre du côté des officiers ( 7 officiers sur 16 sont basques) ainsi qu'à la maistrance (16 officiers mariniers sur 25 sont " du pays "). De même, un petit groupe de seulement 19 matelots basques se retrouve quasiment " dilué " au sein d'un équipage qui compte 125 matelots. L'équipage est complété par 73 volontaires, ce qui correspond au plus faible taux de recrutement de ces hommes parmi les trois corsaires étudiés. L'équipage de La Victoire s'impose donc comme le plus hétérogène des trois étudiés. Enfin, la formation professionnelle des marins basques, et tout particulièrement celle des officiers mariniers, laisse aussi à désirer. L'apprentissage et la formation avant la course sont essentiellement axés sur le cabotage pour les 10 officiers mariniers basques ${ }^{32}$. Le cas de Jean Mongorin $^{33}$ est assez significatif. Entre 1725 et 1744, ce marin originaire de Capbreton et âgé de 57 ans a fondé exclusivement sa carrière sur le cabotage : il a réalisé 48 voyages en barque.

Ainsi, il semble que l'origine géographique de cet équipage et son manque d'expérience permettraient d'expliquer, en partie, la mutinerie déclenchée en son bord à la suite du congédiement de cinq de ses officiers. De fait, ce constat rejoint les conclusions d'André Lespagnol et de Jacques Péret qui avancent l'hypothèse que la loterie corsaire ne se jouerait pas seulement sur mer, mais aussi - et surtout - sur terre ferme, avant même de quitter le port. Chaque équipage corsaire apparaît ainsi comme un micro-

30. Arch. du SHD de Rochefort, 13P8/12, fol. 56 .

31. Ibidem.

32. Arch. du SHD de Rochefort, 13P6/4.

33. Ibid., fol. $7, \mathrm{n}^{\circ} 37$. 
cosme dans lequel cohabitent des individus aux origines géographiques, sociales, culturelles et professionnelles différentes. La comparaison entre nos trois navires amène à considérer que ce brassage doit respecter un certain équilibre sans lequel la croisière corsaire est mise en danger, à l'image de la campagne de la Victoire.

\section{Les gens de mer basques à l'épreuve de la course (1744-1748)}

En mars 1744, l'annonce d'une entrée en guerre maritime et coloniale oblige les armateurs à réorganiser leurs activités. Ainsi, durant la guerre de Succession d'Autriche, 139 armements corsaires sont réalisés au Pays basque (dont 103 à Bayonne) où la course s'impose donc comme l'une des principales activités maritimes entre 1744 et $1748^{34}$. Attirés par des avantages alléchants, les armateurs adoptent cette activité alternative qui constitue un secteur d'emploi très dynamique. La Marine n'y fait pas obstacle, les Basques étant difficiles à mobiliser à Rochefort, le port de guerre le plus proche. La main-d'œuvre se satisfait d'une situation qui permet d'éviter le chômage ou le service du roi, alternatives peu rémunératrices. Les marins ne tardent pas à répondre favorablement ${ }^{35}$. Aussi, qu'ils fassent partie des équipages de La Bellonne, de L'Entreprenante ou de La Victoire, les gens de mer sont systématiquement affectés par ce fait social qui impose son rythme et ses risques.

Les motivations de ces gens de mer pour s'aventurer dans ce type d'expédition sont alors multiples : personnelles (volonté d'échapper au service militaire), financières (hausse des avances versées à l'enrôlement et espoir de butin) ou bien professionnelles (recrutement avantageux). Toutefois, si cette activité propose des avantages non négligeables, le recrutement des hommes n'est pas une tâche aisée pour les capitaines ou armateurs qui voient souvent les meilleurs éléments partir sur les frégates du roi, s'embarquer sur un autre corsaire, ou même choisir les navigations traditionnelles (cabotage, long cours et pêche). Combinées à la pénurie des gens de mer qui affecte la côte atlantique (eu égard à la pression de la Marine sur les cohortes de gens de mer), ces contraintes obligent alors les capitaines à reconsidérer leur technique de recrutement. Ils n'hésitent pas à faire appel aux services d'anciennes recrues, mais surtout, ils élargissent leur cercle de recrutement et " raclent les fonds de tiroir " afin de rassembler un effectif acceptable. De fait, quelles que soient les méthodes de recrutement à la course, la plupart des gens de mer embarqués sur des corsaires connaissent une promotion professionnelle immédiate. La pénurie d'officiers basques assure à la quasi-totalité des officiers mariniers une promotion vers ces fonctions, et cette promotion massive ouvre la mais-

34. Arch. du SHD de Rochefort, 13P8/12 à 16.

35. Voir JAUPART, Fernand, L'activité maritime du port de Bayonne au XVII siècle, Bayonne, Société des sciences, lettres et arts de Bayonne, 1969, qui signale près de 15400 embarquements durant ce conflit. 
trance aux matelots qui libèrent à leur tour des postes pour les volontaires et pour les mousses... Ainsi, une réaction en chaîne provoque presque automatiquement le surclassement des gens de mer les plus méritants et les plus compétents.

Figure 4 - Origine professionnelle des 105 Basques, à leur entrée en course

\begin{tabular}{|l|c|c|c|c|}
\hline $\begin{array}{l}\text { Catégories } \\
\text { de marins }\end{array}$ & $\begin{array}{c}\text { Ascensions } \\
\text { profession- } \\
\text { nelles }\end{array}$ & $\begin{array}{c}\text { Stabilisations } \\
\text { profession- } \\
\text { nelles }\end{array}$ & $\begin{array}{c}\text { Régressions } \\
\text { profession- } \\
\text { nelles }\end{array}$ & $\begin{array}{c}\text { Sans } \\
\text { données }\end{array}$ \\
\hline Mousses & 8 & 9 & 0 & $/$ \\
\hline Matelots & 48 & 16 & 1 & $/$ \\
\hline $\begin{array}{l}\text { Officiers } \\
\text { mariniers }\end{array}$ & 6 & 0 & 0 & 2 \\
\hline Officiers & 4 & 0 & $\mathbf{2}$ & $\mathbf{1 2}$ \\
\hline TOTAL & $\mathbf{6 6}$ & $\mathbf{2 5}$ & $1,9 \%$ & $11,5 \%$ \\
\hline Pourcentage & $62,8 \%$ & $23,8 \%$ & & 10 \\
\hline
\end{tabular}

Au terme de leur expédition corsaire, un premier bilan peut déjà être dressé pour les 105 marins basques étudiés. Si, sur le papier, la course affiche de sérieux atouts pour les nombreux candidats, la réalité est bien plus sombre, voire funeste. Associée à l'idée de loterie, l'issue des campagnes est brutale : c'est quitte ou double. Certains reviennent sains et saufs de leur croisière, tandis que d'autres payent cette prise de risque de leur liberté, si ce n'est de leur vie. Ce cas de figure est bien illustré par les hommes de L'Entreprenante, capturés par les Anglais et emprisonnés dans les geôles anglaises qui avaient la réputation d'être de véritables mouroirs $^{36}$. De même, parmi l'équipage de La Victoire, cinq marins succombent à leurs blessures après l'abordage de La Méditerranée ${ }^{37}$. Enfin, les gens de mer de La Bellonne ne sont pas épargnés par l'épidémie ${ }^{38}$, un mal qui frappe en temps de paix et de guerre. Au final, sur la cohorte de 105 marins basques étudiés, 5 hommes ont péri au cours de la croisière, 4 sont décédés, chez eux, après leur campagne, tandis que 2 autres sont rentrés au pays, au terme de la guerre, mais après une période de captivité dans les prisons anglaises. On réalise aisément que les conséquences de la guerre de course sont avant tout physiques.

Après quelques mois pour certains et de nombreuses campagnes pour d'autres, un premier bilan de l'expérience corsaire peut être dressé quant à l'impact immédiat de cette activité sur la carrière professionnelle des marins. On peut mesurer cet impact en comparant le statut professionnel des gens de mer entre la fin de leur croisière corsaire et leur embarquement

36. Arch. du SHD de Rochefort, 13P6/3 à 5 .

37. Ibid. 13P8/12, fol. 56 .

38. Ibid., 13P8/99. 
suivant, et au moment de leur dernière sortie avant la fin de la guerre, en 1748. Ainsi, parmi les 93 hommes prenant une nouvelle fois le large à la suite de leur première expérience corsaire, seuls cinq ont été rétrogradés au rang de matelots, voire de mousses. En dehors de ces cas, tous les autres sont maintenus dans leur statut et conservent le bénéfice de leur surclassement. La pression sur la ressource humaine est telle que 23 marins de notre cohorte sont promus dès l'embarquement suivant. Pour ces derniers, l'origine sociale ne paraît pas déterminante, puisque les fils de terriens sont largement bénéficiaires ${ }^{39}$. Il semblerait donc que leur promotion dépende plutôt de l'issue de la campagne corsaire.

\section{Figure 5 - Nombre de marins immédiatement promus à la suite des campagnes corsaires étudiées}

\begin{tabular}{|l|c|c|c|}
\hline \multicolumn{1}{|c|}{ Navires } & La Bellonne & La Victoire & L'Entreprenante \\
\hline Marins promus & 13 sur 61 & 9 sur 25 & 1 sur 19 \\
\hline Pourcentage & $21 \%$ & $36 \%$ & $5 \%$ \\
\hline
\end{tabular}

Alors que 22 marins embarqués sur La Bellonne et La Victoire ont aussitôt gravi les échelons vers de plus hautes responsabilités, seul Jean Pirire ${ }^{40}$ (Bayonnais de 34 ans), bosseman à bord de L'Entreprenante, a vu son statut s'élever au rang de chef de prise. Pour tous les autres, la détention dans les geôles anglaises ou encore - en ce qui concerne les officiers - une réputation entachée par un incident disciplinaire semble avoir freiné toute promotion. Toutefois, à côté de ce premier constat, un autre semble se dessiner pour une période d'étude un peu plus longue, qui va de la fin de leur campagne à la signature du traité de paix, en 1748 .

\section{Figure 6 - Nombre de marins promus entre 1744/1745 et la fin de la guerre, en 1748}

\begin{tabular}{|l|c|c|c|c|}
\hline \multicolumn{1}{|c|}{ Navires } & La Bellonne & La Victoire & L'Entreprenante & Total \\
\hline $\begin{array}{l}\text { Nombre de } \\
\text { promus }\end{array}$ & 25 sur 61 & 16 sur 25 & 7 sur 19 & $\mathbf{4 8}$ sur 105 \\
\hline Pourcentage & $41 \%$ & $64 \%$ & $37 \%$ & $\mathbf{4 6} \%$ \\
\hline
\end{tabular}

Au terme du conflit, près de la moitié des gens de mer étudiés ont été promus à un rang supérieur. Cet important résultat atteste du dynamisme de l'activité de course ainsi que de ses conséquences professionnelles pour les marins. En outre, si dans un premier temps les résultats de la croisière corsaire semblent freiner la carrière des gens de mer, les chiffres exposés

39. En effet, sur ces 23 promus, cinq sont fils de terriens, six ont un héritage maritime et l'origine des 12 autres n'est pas renseignée dans les registres matricules.

40. Arch. du SHD de Rochefort, fol. 25, nº 147. 
ci-dessus - notamment ceux concernant les marins de L'Entreprenante démontrent que les résultats d'une campagne n'influencent pas le parcours des navigants sur un plus long terme. Les marins corsaires rentrant d'une chasse déficitaire ne sont pas éternellement marqués par cet échec qui ralentit leur progression durant quelques mois seulement mais leur laisse la possibilité de faire bonne figure et de rattraper leur retard avec des expéditions ultérieures.

La course a donc poussé des hommes parfois sous-qualifiés à détenir du jour au lendemain des postes à responsabilité. Comme nous l'avons précédemment évoqué, les matelots ont fréquemment été embarqués en tant qu'officiers mariniers lors des campagnes corsaires. C'est pour ces marins une formation accélérée et rude qui a laissé, en cas de réussite, une porte ouverte aux plus hauts grades. Parfois, certains individus renouvelant d'honnêtes performances ont vu leur carrière décoller. Ainsi, d'après le graphique ci-dessous, on remarque que parmi les 46 gens de mer recrutés en tant qu'officiers mariniers lors de leur entrée en course - et qui ont été pour la plupart matelots avant cet événement - trois sont morts ou ont abandonné entre-temps, 27 ont gardé leur statut jusqu'au terme du conflit, et enfin 16 ont été promus au rang des officiers avant la fin de la guerre, en 1748.

La course influence donc bel et bien la vie des gens de mer, à l'image de l'échantillon des 105 marins basques étudiés. À partir du moment où ils empruntent cette voie, la plupart de ces hommes voient leur carrière professionnelle prendre un nouveau souffle, en très peu de temps. Cela conforte la course dans son statut d'accélérateur professionnel, que ce soit dès le recrutement ou même au cours des croisières qui s'enchaînent. Bien que ces promotions concernent en premier lieu les membres de l'état-major, de nombreuses avancées sont à noter, même si les marins connaissent l'échec durant leur campagne. Ils ne sont, finalement, pénalisés que le temps d'une croisière. A minima, le marché du travail permet aux corsaires leur recrutement au poste précédent, et une telle stagnation prend fin à l'issue de la croisière suivante. Au regard de toutes ces observations, la course favorise donc, sur un temps court ou immédiat, la carrière des 105 gens de mer basques, sur les plans physique, psychologique, financier et professionnel.

\section{L'après-guerre humain d'un port de commerce secondaire (1749-1755)}

Négociés et débattus durant plusieurs semaines par les diplomates, les traités d'Aix-la-Chapelle offrent aux belligérants une paix sans vainqueur (1748). À l'annonce de la paix, l'effort de guerre est stoppé, les hommes reviennent dans leurs foyers et toute activité conflictuelle cesse. Pour beaucoup, la signature de ces traités rime avec reconversion, que ce soit pour les gens de mer qui doivent à nouveau envisager leurs migrations tempo- 
raires $^{41}$ vers les ports de Bordeaux ${ }^{42}$, La Rochelle ${ }^{43}$ ou Nantes ${ }^{44}$, ou pour le marché du travail qui doit prendre en compte les avancées professionnelles survenues durant la guerre. En effet, au terme de ce conflit, nombre de matelots ont été promus au rang d'officiers mariniers, alors qu'en parallèle, la proportion de marins rentrés du service ayant bénéficié de telles promotions est bien moindre ${ }^{45}$. Après 1749 , le corps de la maistrance a considérablement été étoffé; c'est là l'une des conséquences de l'ascension professionnelle occasionnée par la course. De fait, face à la saturation du corps des officiers mariniers, il conviendrait de se demander si les bénéfices suscités par la course n'ont pas été qu'éphémères et ne résulteraient pas uniquement d'un contexte particulier.

\section{Figure 7 - Évolution professionnelle des marins basques entre 1744 et 1748}

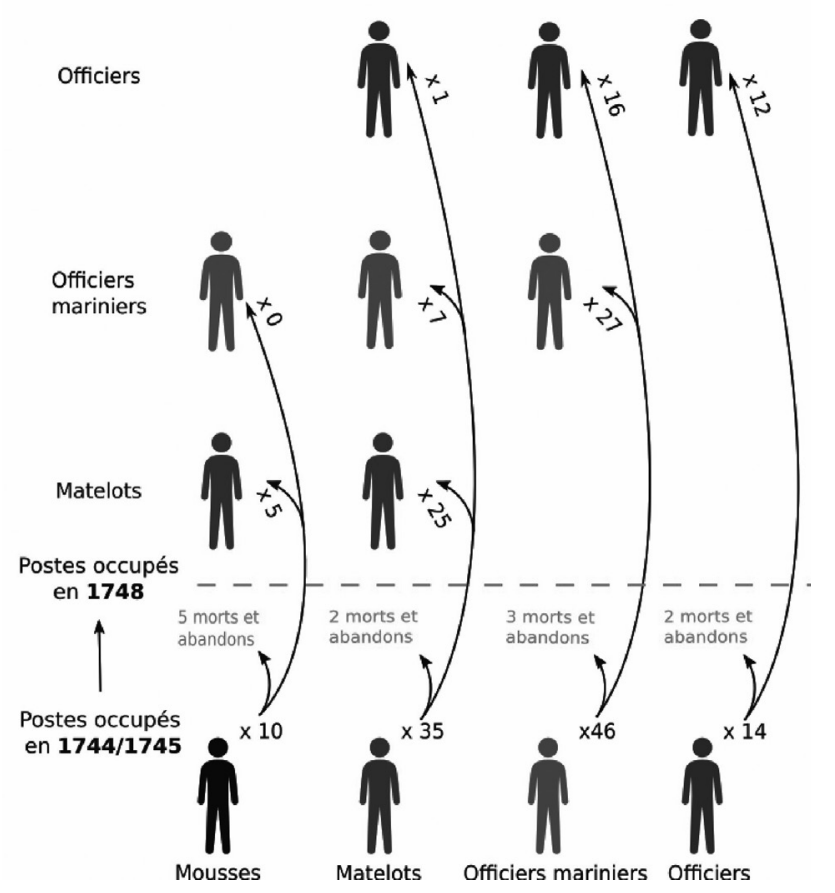

41. Arch. du SHD de Rochefort, 13P6/8 à 10.

42. ACERRA, Martine et MEYER, Jean, La grande époque de la marine à voile..., op. cit., p. 86.

43. PÉRET, Jacques, Les corsaires de l'Atlantique..., op. cit.

44. MEYER, Jean, L'armement nantais dans la seconde moitié du XVII ' siècle, Paris, SEVPEN, 1969.

45. Arch. du SHD de Rochefort, 13P6/8 à 10. 
Figure 8 - Évolution professionnelle de 43 marins basques entre 1743 et 1755

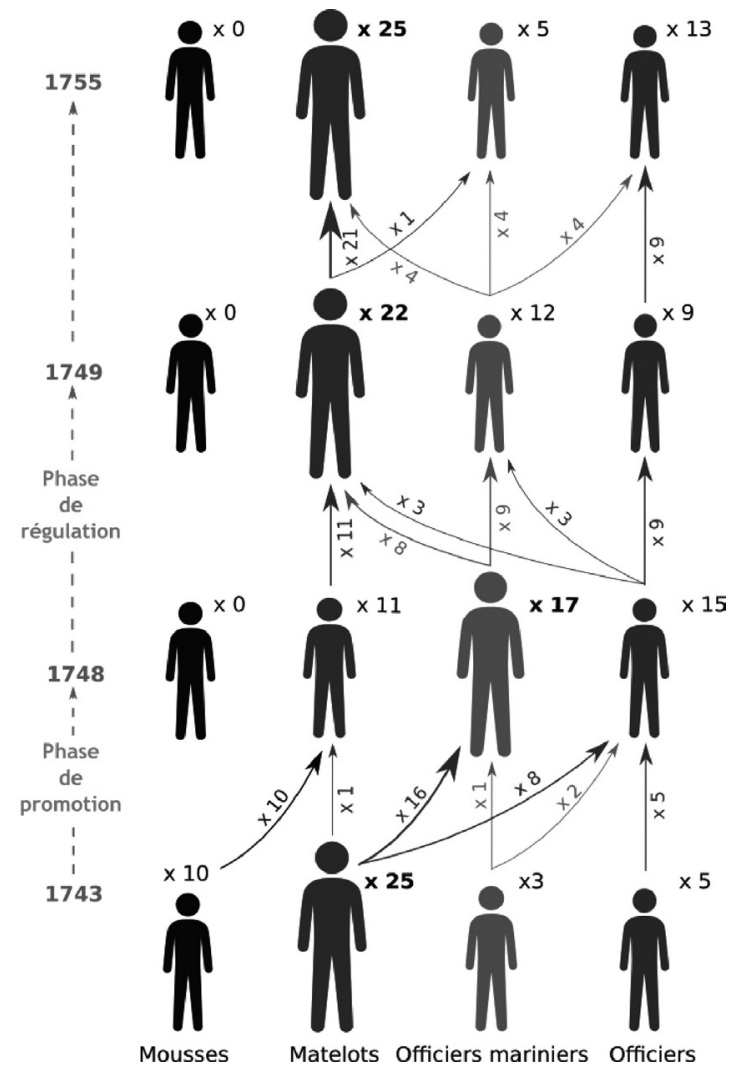

Sans égard pour les origines sociales ou les navigations faites par les marins, la course offre une accélération professionnelle sur un temps court, celui de la guerre. En revanche, sur le long terme - c'est-à-dire jusqu'à la veille de la guerre de Sept Ans, en 1755 - cette argumentation paraît remise en cause. Afin d'étudier l'évolution professionnelle sur ce temps long, il paraît légitime de se pencher sur le parcours des 43 marins basques ayant survécu jusqu'à la veille de cette nouvelle guerre. Parmi eux, la très grande majorité - 40 - se trouve alors bénéficiaire d'une promotion à la faveur du conflit, pour les mêmes raisons que celles qui ont été précédemment évoquées. Toutefois, pour la plupart de ces hommes, une fois la guerre terminée, la transition professionnelle ne correspondait pas à ce que laissait présager la course.

$\mathrm{Au}$ regard des données exposées ci-dessous, l'activité corsaire a, certes, promu de nombreux marins vers de plus hautes responsabilités, mais ces évolutions se sont révélées de circonstance. Alors que la catégo- 
rie des matelots prédomine numériquement à la veille de la guerre, avec 25 hommes, parmi les 43 Basques étudiés, cette domination numérique s'estompe durant le conflit, avant de retrouver, après-guerre, un statu quo ante bellum presque parfait. Cela montre l'existence d'une vague de déclassements au terme du conflit, et un évident retour en arrière pour nombre de gens de mer.

En effet, sur ce même contingent de 43 Basques, 12 mousses et matelots ont vu leur évolution de carrière arrêtée net une fois la paix revenue. Pour 15 autres, cette activité paraît plus bénéfique puisqu'ils ont quand même gravi les échelons, à court ou à moyen terme. Dès lors, ils investissent les rangs des officiers ou des plus hautes fonctions de la maistrance. Enfin, 16 marins ont subitement vu leur statut professionnel régresser, quelle que soit leur trajectoire ou encore leur participation à l'une des croisières étudiées. Ainsi, pour les deux tiers des marins de notre échantillon, la course n'est pas un facteur d'accélération professionnelle, mais offre plutôt une progression illusoire, à l'image du parcours de Bernard Larrebat, passé successivement aux grades de mousse, matelot, bosseman, contremaître puis maître d'équipage avant de redevenir matelot après la guerre ${ }^{46}$.Dans la mesure où les marins qualifiés manquent dans les ports basques, les armateurs n'hésitent pas à recruter les gens de mer pour la course à un poste qui est, en réalité, au-dessus de leurs compétences. Une fois la paix revenue, Bayonne redevient un port de commerce secondaire et les hommes repartent vers Bordeaux, havre tentaculaire, dans lequel le marché du travail est saturé d'officiers mariniers. Toutefois, entre ceux qui ont fait leurs preuves à la course et ceux qui ont persévéré à la droiture pendant la guerre, il y a une différence de taille. Dès lors, les anciens corsaires se retrouvent probablement en situation de devoir batailler pour maintenir leur grade acquis un peu trop rapidement durant le conflit, du point de vue de la profession. Malheureusement, pour une grande majorité d'entre eux, les promotions hâtives de la course font suspecter en arrière-plan des lacunes professionnelles, dont la conséquence paraît sans appel : le déclassement. Cela représente un retour en arrière pour 16 marins, comme en témoigne la régulation du corps des matelots après 1748. Sous cet angle, les ascensions provisoires procurées par la course répondent clairement à un contexte exceptionnel.

De prime abord, pour les deux tiers des marins étudiés, la course n'a pas profité à leur carrière. Aussi, afin d'observer l'impact de l'expérience corsaire sur la carrière professionnelle du tiers restant, il paraît légitime de comparer leur parcours avec celui des marins de la Seudre, étudiés par Thierry Sauzeau pour la fin du XvIII ${ }^{\mathrm{e}}$ siècle. Entre Bordeaux et La Rochelle, pour ce qui regarde le marché du travail, les marins de la Seudre, situés à proximité immédiate de Rochefort et de la Marine royale, ignorent la course à l'époque où les Basques s'en font une spécialité.

46. Arch. du SHD de Rochefort, 13P6/1, fol. 39, n 338; 13P6/4, fol. 70, n 415; 13P6/8, fol. $8, \mathrm{n}^{\circ} 48$. 
Le parcours des 43 Basques constituant la cohorte de l'après-guerre de Succession d'Autriche révèle 15 promotions vers l'élite de la navigation. Trois deviennent maîtres de cabotage. Ils ont en commun d'avoir débuté leur carrière comme mousses, à l'âge de 12 ans, soit un profil voisin de celui de leurs collègues de la Seudre qui affichaient un âge moyen de 14 ans lors de leur entrée en apprentissage ${ }^{47}$. Après avoir axé leur carrière maritime sur la barque, ces trois Basques ont passé avec succès leur examen de maître au cabotage à l'âge moyen de 30 ans, tandis que leurs homologues charentais obtenaient cette qualification dès 25 ans. Bien que les candidats à cet examen ne soient que très peu représentatifs de l'ensemble des marins basques, une remarque apparaît : si la course accélérait vraiment la carrière des gens de mer, les trois Basques auraient sans nul doute été promus dès leur vingt-cinquième année. En réalité, pour les marins du cabotage, l'expérience corsaire n'a été d'aucun secours sur le plan de l'avancement, et on peut même avancer qu'elle l'a retardé. Si l'on se penche sur le contingent de Basques accédant aux plus hauts grades du bord après 1748, le rôle d'accélérateur de la course n'est pas plus net. En effet, les Basques accédaient aux postes d'officiers d'état-major vers la trentaine, en même temps que les marins de la Seudre à la même époque ${ }^{48}$.

Figure 9 - Promotion des cinq marins basques aux postes de commandement après la guerre

\begin{tabular}{|l|c|c|c|c|c|}
\hline & $\begin{array}{c}\text { Balanqué } \\
\text { Antoine }\end{array}$ & $\begin{array}{c}\text { Vergez } \\
\text { Jean }\end{array}$ & $\begin{array}{c}\text { Perdigau } \\
\text { Jacques }\end{array}$ & $\begin{array}{c}\text { Gignan } \\
\text { Pierre }\end{array}$ & $\begin{array}{c}\text { Rivière } \\
\text { Léon }\end{array}$ \\
\hline $\begin{array}{l}\text { Origine } \\
\text { socio-profes- } \\
\text { sionnelle }\end{array}$ & $\begin{array}{l}\text { Capitaine } \\
\text { de navire }\end{array}$ & Rentier & Cordonnier & Armurier & Matelot \\
\hline $\begin{array}{l}\text { Plus haute } \\
\text { fonction } \\
\text { atteinte }\end{array}$ & $\begin{array}{c}\text { Capitaine } \\
\text { en second }\end{array}$ & Capitaine & $\begin{array}{c}\text { Capitaine } \\
\text { en second }\end{array}$ & $\begin{array}{c}\text { Capitaine } \\
\text { en second }\end{array}$ & Capitaine \\
\hline $\begin{array}{l}\text { Age de } \\
\text { l'obtention } \\
\text { de cette } \\
\text { fonction }\end{array}$ & 28 ans & 30 ans & 33 ans & 29 ans & 30 ans \\
\hline
\end{tabular}

En dépit de la pénurie de marins, liée aux décès, à la captivité ou à la mobilisation des gens de mer durant le conflit, l'Amirauté n'a pas facilité les ascensions aux plus hauts grades, puisque parmi les 105 marins initialement étudiés, seuls deux hommes sont finalement parvenus à investir la plus haute distinction de la navigation : l'examen de capitaine. Ainsi, la période de guerre et l'activité corsaire n'ont pas fabriqué les élites et n'ont

47. SaUzeau Thierry, Les marins de la Seudre..., op. cit., p. 216.

48. Ibid., p. 199-204 et BuTI, Gilbert, CABANTOus, Alain, Etre marin en Europe occidentale : 1550-1850, Rennes, PUR, 2016, p. 187. 
pas accéléré la carrière professionnelle de l'échantillon de marins étudié. Finalement, la sueur, la fatigue, l'intégration à un réseau, la réputation et la chance bâtissaient plus sûrement la carrière d'un futur officier ou d'un maître, que l'appel d'air temporaire offert par le marché du travail très particulier de la course, dont les promotions professionnelles ont rarement été confirmées une fois la paix revenue.

Si l'on doit dresser un bilan de l'impact de l'expérience corsaire sur la vie des gens de mer, celui-ci ne peut être que très mitigé, partagé entre opportunités et illusions. Au fil de cette étude, les croisières de La Bellonne, de L'Entreprenante et de La Victoire ainsi que les parcours de leurs 105 marins basques dévoilent une image nuancée. La course offre finalement une image moins exaltante que ne le laissaient présager les écrits d'Édouard Ducéré. D'abord, conformément aux analyses menées par André Lespagnol ou Jacques Péret, le recrutement corsaire des marins basques a dû respecter un certain équilibre, sans quoi la croisière pouvait avoir de fâcheuses conséquences. Certes, la course était soumise au hasard et à la chance mais la composition de l'équipage permettait de nourrir certains espoirs, ou faisait naître des craintes, quant à l'issue de la croisière.

Au-delà de cette organisation, nombre de gens de mer, plus ou moins compétents, se sont portés candidats. Hausses de salaire, échappatoires au service, perspectives d'ascension professionnelle ou bien espoirs de mettre la main sur un butin important devaient jouer un rôle dans l'attractivité de l'activité corsaire. Mais la réalité a souvent été tout autre. La mort, les combats, les naufrages, les maladies et la captivité en ont retenu plus d'un. Pour les moins chanceux, la course s'est payée au prix de la liberté, voire de la vie. D'autres sont revenus de leur campagne sains et saufs et ils ont pu profiter des bénéfices de la course. En effet, l'activité corsaire a aussi été synonyme d'accélération et d'ascension professionnelle sur un temps court, et ce à différents niveaux. Certains se sont vus promus dès avant l'embarquement, d'autres l'ont été dès l'expédition suivante, voire même, jusqu'à la fin de la guerre (1748) pour 48 d'entre eux, soit près de la moitié de la cohorte étudiée. Dès lors, un premier constat s'impose : les gens de mer du Pays basque qui choisissaient la course avaient une chance sur deux de gravir les échelons de la carrière sans tarder. Dans un contexte de pénurie de main-d'œuvre qualifiée, les recruteurs fermaient aisément les yeux sur leur qualification médiocre et ils leur ont offert l'opportunité d'une promotion.

Au regard de ces éléments, une conclusion se dégage. La course a bien eu des impacts " positifs " sur la carrière professionnelle des gens de mer du Pays basque durant la guerre de Succession d'Autriche, en ce sens qu'elle leur a offert des promotions de carrière. Mais ces promotions sont la plupart du temps demeurées temporaires. En effet, à l'annonce de la signature des traités de paix d'Aix-la-Chapelle, les statuts des marins ont été réévalués, généralement à la baisse. Parmi les 43 marins de la cohorte 
initiale dont les parcours maritimes ont encore pu être retracés après la guerre, seuls 15 sont parvenus à maintenir le grade décroché durant le conflit. Pour ce qui est des 28 autres, ils ont été rétrogradés, chez les matelots en majorité. L'idée même que la course produisait et accélérait l'ascension professionnelle doit donc largement être nuancée. En témoignerait par exemple la comparaison des évolutions de carrière entre les Basques et les marins de la Seudre. Finalement, les avantages offerts par la course n'ont été que temporaires et illusoires.

Pour autant, cela n'a pas empêché les 25 marins naviguant toujours à l'aube de la guerre de Sept Ans, de réitérer l'expérience corsaire. Une sorte de réflexe corsaire a donc resurgi à l'annonce d'une nouvelle guerre pour ces hommes souhaitant en découdre, soit qu'ils aient été alors dans la nécessité financière soit qu'ils aient été mus par le simple respect de la tradition. Pour ces marins désireux de retenter leur chance, la roue de la loterie corsaire allait pouvoir de nouveau tourner.

\section{RÉSUMÉ}

L'ouverture de la guerre de Succession d'Autriche au front maritime (1744) a conduit de nombreux armateurs et gens de mer basques à se convertir à la course dans l'espoir de trouver emploi, gloire et fortune. À partir du moment où ils s'aventurent dans ces croisières, les acteurs de ce fait social sont inévitablement confrontés à une " loterie corsaire " (André Lespagnol) qui leur impose des mutations d'ordre professionnel, financier ou encore physique. L'étude des carrières de trois équipages corsaires basques, analysées en amont et en aval de leurs croisières, témoigne d'une activité synonyme d'ascensions et accélérations professionnelles durant la guerre. Mais le retour à la paix (1748) rebat les cartes et laisse entrevoir que les avantages acquis en temps de guerre sont souvent temporaires et illusoires.

\section{ABSTRACT}

The beginning of the Austrian Succession War on the seafront (1744) led Basque shipowners and seafarers to convert to privateering in order to find employment, glory and fortune. Subsequently, from the moment they ventured into such expeditions, the actors of this social phenomenon were inevitably confronted by the "privateer's lottery" (André Lespagnol), which entailed professional, financial and physical changes. Among them, the careers of three Basque privateer crews, analysed upstream and downstream of their expeditions, exemplifies how the activity became synonymous of professional ascent and success, during wartime. However, the return to peace (1748) led to profound changes and suggests that the benefits accrued were often temporary and illusory. 